\title{
Análisis de la relación de las universidades con sus stakeholders en Internet
}

\author{
Gaete Quezada, Ricardo*
}

\section{Resumen}

El presente articulo tiene como objetivo analizar la relación de universidades españolas y chilenas con su stakeholders en internet, para la cual en primer lugar se identificaron los tipos de stakeholders (según Comisión Europea, 2008) y posteriormente se revisan los contenidos de dos sitios web de las universidades diseñados en términos de estas relaciones. El método de investigación empleado fue el estudio de casos mediante el cual se compara la relación que una muestra de universidades en internet españolas y chilenas mantienen con sus stakeholders (partes interesadas en Internet) a través de un análisis de contenidos de sus sitios web, considerando tres tipos en la web: información, comunicación y procesos estratégicos. Los resultados indican que no existen diferencias significativas en los contenidos que se relacionan con información institucional, pero en lo que se refiere a los procesos estratégicos y especialmente en cuanto a los contenidos asociados con la comunicación en Internet, las diferencias son importantes. Además, permiten inferir una importante presencia de la transparencia y acceso a la información por parte de la mayoría de las universidades estatales de la muestra, así como una relación con las partes interesadas o grupos de interés de tipo contractual o transaccional, debido al bajo desarrollo o presencia de contenidos de comunicación que proponen una relación más de carácter relacional.

Palabras clave: Stakeholders o partes interesadas, gestión universitaria, sitios Web, análisis de contenidos, educación superior.

\section{Analysis of the Relationship between Universities and their Stakeholders on Internet}

\author{
Abstract \\ The objective of this article is to analyze the relationship between Spanish and Chilean univer- \\ sities and their stakeholders on Internet. To accomplish this, in the first place, the types of stakehold- \\ ers were identified (according to the European Commission, 2008); later, the contents of two univer- \\ Recibido: 03-02-11. Aceptado: 08-11-11 \\ * Académico Jornada Completa del Departamento de Ciencias Sociales de la Universidad de \\ Antofagasta. Magíster en Gestión Pública y Licenciado en Ciencias de la Administración. \\ E-mail: rgaete@uantof.cl
}


sity websites designed in terms of these relationships are reviewed. The research method was case studies, through which it was possible to compare the relationship maintained with the stakeholders (parties interested in the Internet) through content analysis of their websites, considering three types of web content: information, communication and strategic processes. Results indicate that there are no significant differences in content related to institutional information, but regarding strategic process and, especially, in terms of content associated with communication by Internet, the differences are significant. Furthermore, the results permit inferring a significant presence of transparency and access to information by the majority of the state universities in the sample, as well as a relationship with the interested parties or interest groups of a contractual or transactional type, due to the low development or presence of communication content that proposes a closer relationship.

Keywords: Stakeholders or interested parties, university management, websites, content analysis, higher education.

\section{Introducción}

Uno de los desafíos que actualmente deben enfrentar las universidades es la creciente necesidad de demostrar que efectivamente su funcionamiento y razón de ser se orientan a la satisfacción de las necesidades y problemáticas de las personas, grupos o instituciones con los que interactúan.

En el logro de ese propósito, la teoría de los stakeholders se presenta como una interesante oportunidad de valorar el grado de relación y/o vinculación existente entre la universidad y las distintas partes interesadas con las cuales estas instituciones se vinculan.

A su vez, los procesos relacionales que establecen las organizaciones en la actualidad, se orientan cada vez más a la utilización del espacio virtual para su desarrollo, situación que pone de manifiesto la necesidad de diseñar sitios web que faciliten la interacción de la organización con sus stakeholders (partes interesadas).

De esta manera, el propósito de este estudio es doble, por una parte, identificar los stakeholders con los cuales dichas universidades relacionan su queha- cer, y su convergencia con la tipología propuesta por la Comisión Europea (2008), y por la otra, analizar los contenidos de los sitios web que las universidades de la muestra utilizan para relacionarse con sus partes interesadas.

En este contexto, el artículo emplea la metodología del estudio de caso para constatar las relaciones que una muestra de universidades chilenas y españolas mantiene con sus stakeholders en Internet, utilizando la técnica de análisis de contenidos para examinar las páginas, contenidos y enlaces existentes en cada sitio web.

En el desarrollo del estudio, se definieron tres categorías de análisis de los sitios web de las universidades de la muestra: información, comunicación y procesos estratégicos. Cada categoría posee una serie de contenidos que fueron revisados a través de la página de inicio, buscador del sitio y mapa web.

Los principales resultados señalan que ambos grupos de universidades muestran una mayor presencia de contenidos en las categorías información y procesos estratégicos, siendo menor el desarrollo en lo relacionado con la comuni- 
cación 2.0, especialmente en el caso de las universidades chilenas y las universidades privadas de Castilla y León, donde la incorporación de blogs institucionales, podcast o foros es muy baja o inexistente.

Con estos resultados, se puede afirmar desde una perspectiva exploratoria descriptiva que las universidades analizadas desarrollan a través de sus sitios web una relación mayoritariamente uni-direccional, contractual y de carácter transaccional con sus stakeholders, existiendo un bajo desarrollo de relaciones en Internet que permitan configurar redes y comunidades de aprendizaje recíproco entre las universidades y sus partes interesadas.

\section{La teoría de los stakeholders}

La primera sistematización de una teoría de los stakeholders se encuentra relacionada con los aportes de R. Edward Freeman en 1984, quién desde la gestión estratégica de las organizaciones elabora esta teoría (Donaldson \& Preston, 1995; Lozano, 1999; Post et al., 2002; Matten et al., 2003; Sison, 2008; Lozano, 2009).

De acuerdo con Freeman (1984: 53) el término stakeholder permite identificar a "cualquier grupo o individuo que pueda afectar o se vea afectado por la consecución de los propósitos de la empresa". Además, identifica como los stakeholders de una empresa a: clientes, dueños, competencia, proveedores, gobiernos, medios de comunicación, empleados, defensa del consumidor, ambientalistas y organizaciones comunitarias locales.

A pesar de la simpleza de la definición aportada por Freeman, la identificación de un stakeholder por parte de una organización puede adquirir una comple- jidad importante, debido a las múltiples formas, características y definiciones que los stakeholders pueden adquirir, lo que muchas veces dificulta a la organizaciones tener claridad de quienes son estos grupos o individuos que son partes interesadas en su quehacer (Lozano, 1999; Hax, 2006; Prandi, 2007; Setó, 2007).

Algunos criterios susceptibles de utilizar para identificar y clasificar a los stakeholders, más allá de la confección de simples listados, nos colocan ante dos grandes perspectivas para identificar a las partes interesadas de una organización (Lozano, 1999; Moneva, 2005; Marín, 2008):

a) Aquellos grupos de interés internos (directivos, empleados y accionistas, por ejemplo).

b) Aquellos de carácter externo. (clientes, proveedores, administraciones públicas, entidades financieras, medio ambiente, entre otros).

Sin embargo, de acuerdo con Clarkson (1995) y Marín (2008) los stakeholders también pueden clasificarse en dos categorias: (1) aquellos que poseen un carácter primario (alta dirección, recursos humanos, accionistas / inversores, proveedores, clientes, competencia); (2) aquellos grupos de carácter secundario. (Entorno social, entorno ambiental, administraciones públicas).

Además, es habitual encontrarse con que las organizaciones identifican como sus stakeholders solamente a aquellos grupos que se encuentran organizados, como un sindicato por ejemplo, o aquellos grupos con los cuales la organización mantiene algún tipo de relacional contractual (Post et al., 2002; Antonacopoulou \& Meric, 2005; Hax, 2006). 
Así, existen en la literatura diversas tipologías de stakeholders que buscan delimitar su identificación desde perspectivas más bien genéricas y restringidas (Freeman, 1984; Carroll, 1991; Donaldson \& Preston, 1995; Agle et al., 1999; Comisión Europea, 2001), hasta las tipologías más extendidas y con una orientación más reticular para identificar a las partes interesadas de una organización (Post et al., 2002; Ferrary, 2005; Durán, 2006; Werther \& Chandler, 2006). El Cuadro 1 ofrece un resumen de las tipologías antes señaladas y su grado de convergencia en cuanto al tipo de stakeholder.

Como puede observarse, los stakeholders que se repiten en las nueve tipologías analizadas en este estudio son los empleados o trabajadores y los clientes, usuarios o consumidores. Por el lado contrario, las partes interesadas que ofrecen una menor presencia en las tipologías son los partidos políticos, las generaciones futuras y las asociaciones profesionales, estas dos últimas provenientes de tipologías extendidas.

De esta manera, la gestión de la relación con los stakeholders se vincula con la creación de valor o riqueza generada por la organización para las partes interesadas y para sí misma (Post et al., 2002, 2002b; Antonacopoulou \& Meric, 2005; Ferrary, 2005; Torres, 2006; Setó, 2007), siendo relevante considerar que el valor creado es limitado, y que por lo tanto se generará una competencia entre todas las partes que están directa e indirectamente involucradas en el proceso de creación de valor, por lo que la organización debe ser capaz de gestionar las interacciones y disputas que puedan generarse en este proceso.
En este último aspecto, Ferrary (2005) recuerda que en un sistema stakeholder de relaciones entre la organización y sus partes interesadas, los distintos grupos o actores no sólo interactúan de manera uni y bi-direccional con la organización, sino que regularmente interactúan también con las otras partes interesadas en forma de red, con lo que pueden lograr intervenir directamente con mayor fuerza en el funcionamiento de la organización, perspectiva reticular de la relación con los stakeholders compartida por otros autores tales como Lozano $(1999,2009)$ o Post et al. (2002a, 2002b).

En cuanto a la aplicación de la teoría de los stakeholders en el ámbito de la Educación Superior, la Comisión Europea (2008) en el análisis de la gestión y buen gobierno de las universidades europeas, señala que es posible identificar partes interesadas externas entre las que se destaca al gobierno central, regional o local, los empleadores en el mercado de trabajo, sindicatos, asociaciones de estudiantes, representantes de la sociedad civil, los graduados, los padres de los estudiantes.

En cuanto a los stakeholders internos de las universidades, la Comisión Europea identifica entre otros a: los órganos de gobierno institucional, y las personas empleadas o matriculados en una institución de educación superior.

Asimismo, Burrows (1999) propone una tipología de stakeholders para las universidades europeas, sin distinguir entre internos o externos pero agrupándolos en 12 categorías que cuentan con sus respectivos grupos constitutivos: entidades gobernantes, órganos de administración universitaria, empleados, clientes, proveedo- 


\section{Cuadro 1}

Tipologías de stakeholders en la literatura

\begin{tabular}{|c|c|c|c|c|c|c|c|c|c|c|}
\hline & & Tip & gías $\mathrm{g}$ & néric & & & logía & exte & lidas & \\
\hline & 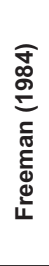 & 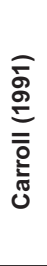 & 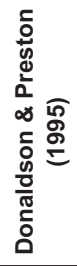 & 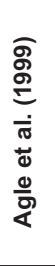 & 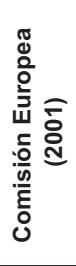 & 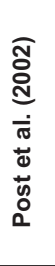 & 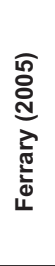 & 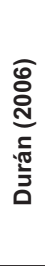 & 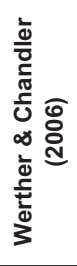 & సँّ \\
\hline Empleados, trabajadores & $x$ & $x$ & $x$ & $x$ & $x$ & $x$ & $x$ & $x$ & $x$ & 9 \\
\hline $\begin{array}{l}\text { Directivos, Gobierno } \\
\text { Corporativo }\end{array}$ & & & & & & & $\mathrm{x}$ & & $\mathrm{x}$ & 2 \\
\hline $\begin{array}{l}\text { Accionistas, propietarios } \\
\text { o dueños }\end{array}$ & $\mathrm{x}$ & $\mathrm{x}$ & & $\mathrm{x}$ & $x$ & $\mathrm{x}$ & $\mathrm{x}$ & $\mathrm{x}$ & $\mathrm{x}$ & 8 \\
\hline Sindicatos & & & $x$ & & & $x$ & $x$ & & $x$ & 4 \\
\hline $\begin{array}{l}\text { Clientes, consumidores } \\
\text { o usuarios }\end{array}$ & $\mathrm{x}$ & $\mathrm{x}$ & $\mathrm{x}$ & $\mathrm{x}$ & $x$ & $\mathrm{x}$ & $x$ & $\mathrm{x}$ & $\mathrm{x}$ & 9 \\
\hline Acreedores o inversionistas & & & $x$ & & & & $x$ & $x$ & $x$ & 4 \\
\hline Proveedores y distribuidores & $x$ & $x$ & $x$ & & $x$ & & $x$ & $x$ & $x$ & 7 \\
\hline Comunidad local & $\mathrm{x}$ & $\mathrm{x}$ & $\mathrm{x}$ & $x$ & & $x$ & & $\mathrm{x}$ & $\mathrm{x}$ & 7 \\
\hline $\begin{array}{l}\text { Gobierno Nacional } \\
\text { o Regional }\end{array}$ & $\mathrm{x}$ & & $\mathrm{x}$ & $\mathrm{x}$ & $\mathrm{x}$ & $\mathrm{x}$ & $\mathrm{x}$ & $x$ & $\mathrm{x}$ & 8 \\
\hline $\begin{array}{l}\text { Organizaciones sin fines } \\
\text { de lucro }\end{array}$ & & $\mathrm{x}$ & & & & $\mathrm{x}$ & & & $\mathrm{x}$ & 3 \\
\hline ONG & $x$ & & & & $x$ & & & & $x$ & 3 \\
\hline $\begin{array}{l}\text { Entes reguladores, } \\
\text { Adm. Pública }\end{array}$ & & & & & & $\mathrm{x}$ & & $\mathrm{x}$ & $\mathrm{x}$ & 3 \\
\hline Medio ambiente & & & & & $x$ & & & & $x$ & 2 \\
\hline Partidos políticos & & & $x$ & & & & & & & 1 \\
\hline Socios comerciales & & & & & $x$ & $x$ & & & & 3 \\
\hline Competencia & $x$ & $x$ & & & & & $x$ & & & 3 \\
\hline Medios de comunicación & $x$ & & & & & & $x$ & $x$ & & 3 \\
\hline Generaciones futuras & & & & & & & & $x$ & & 1 \\
\hline Asociaciones profesionales & & & & & & & $x$ & & & 1 \\
\hline Asociaciones de clientes & $\mathrm{x}$ & & & & & & $x$ & & & 2 \\
\hline
\end{tabular}

Fuente: Elaboración propia con base en autores señalados (2010). 
res, competidores, donantes, comunidad local, organismos reguladores gubernamentales, organismos reguladores no gubernamentales, Intermediarios financieros, socios de joint ventures.

Según Burrows (1999), resulta insuficiente que las universidades únicamente identifiquen quiénes son sus partes interesadas, situación que no asegura una adecuada comprensión y entendimiento de los intereses y demandas que dichos stakeholders plantean a la universidad, lo cual puede transformarse en focos de conflicto en el futuro. En razón de estas deficiencias, esta autora identifica 4 dimensiones para diferenciar las partes interesadas según sus intereses y demandas, con el propósito de distinguir adecuadamente la forma de gestionar dicha relación: posición, grado de participación, potencial para cooperación o amenaza, y su interés e influencia en la organización.

Dentro de este apretado repaso a la teoría de los stakeholders, también es posible encontrar en la literatura algunas experiencias empíricas de su utilización a nivel universitario, como en el caso de los trabajos de Caballero et al. (2007, 2009) quienes utilizan esta teoría para analizar la empleabilidad de los egresados de las universidades españolas. En Gaete (2010), se desarrolla un análisis de las declaraciones de misión y visión para identificar los discursos dirigidos hacia los stakeholders de las universidades de la macro zona norte de Chile, utilizando para ello una tipología de partes interesadas de carácter genérico.

\section{Tipología de los stakeholders relacionados con las universidades}

a) Universidades de Castilla y León

En relación con los resultados obtenidos de la revisión de los sitios web de esas instituciones respecto de la identificación de las partes interesadas propuestas por la Comisión Europea (2008), se tiene que dichas universidades se orientan mayoritariamente hacia el ámbito interno, especialmente en el caso de las universidades públicas. Los resultados se resumen en el Cuadro 2.

Las universidades públicas de Castilla y León (en adelante CyL) muestran levemente un mayor grado de convergencia con la tipología de partes interesadas propuesta por la Comisión Europea (2008), basado fundamentalmente en la mayor orientación de sus sitios web hacia los sindicatos y asociaciones de estudiantes.

Además, se observa que son las partes interesadas internas de la tipología analizada las que reciben el mayor número de referencias en los contenidos web de cada sitio examinado, con la sola excepción de la Universidad Pontificia de Salamanca que no muestra vínculos para ningún stakeholder interno, sólo registra enlaces y contenidos específicos para los ex alumnos.

Finalmente, los graduados o titulados, gobierno universitario y estudiantes son las partes interesadas que más presencia tienen en la sub-muestra, ya que siete universidades los reconocen como stakeholders relevantes a los cuales destinan enlaces y contenidos en sus sitios web. 


\section{Cuadro 2}

\section{Partes interesadas de las universidades de Castilla y León}

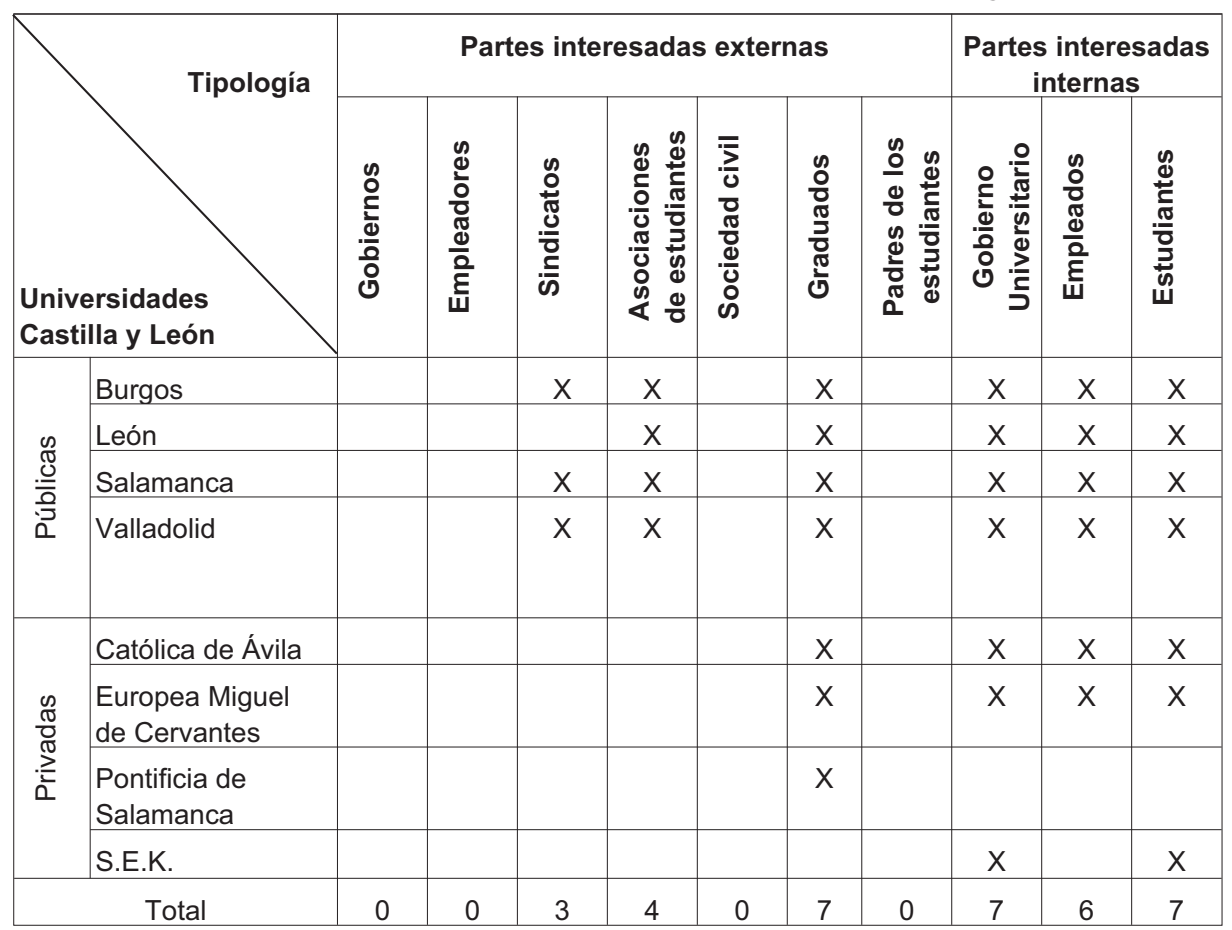

Fuente: Elaboración propia (2010) con base en la Comisión Europea (2008).

Otra diferencia importante que se observa es que ninguna de las universidades privadas de CyL ofrece enlaces para sindicatos o asociaciones de estudiantes, situación totalmente inversa en las universidades públicas de la Comunidad, es una señal clara de la importancia que se les otorga a estos stakeholders, quedando, a su vez, demostrado por su participación en el gobierno universitario (ver Gráfico 1).

Además de las partes interesadas de la tipología analizada, existen otros grupos o instituciones que poseen enlaces en los sitios web de las universidades de CyL, tales como futuros estudiantes (Burgos, León, Miguel de Cervantes), preuniversitarios (Valladolid), fundaciones (Valladolid, Salamanca), conferencia de rectores (Valladolid), colegios e institutos de educación secundaria (Miguel de Cervantes) y patrocinadores (Valladolid), entre otras partes interesadas.

b) Universidades macro zona norte de Chile.

El segundo caso de estudio es el de las universidades de la macro zona norte de Chile (en adelante MZN), donde es importante señalar que la mayoría de las instituciones que componen esta sub- 


\section{Gráfico 1}

\section{Distribución de universidades de Castilla y León por stakeholder}

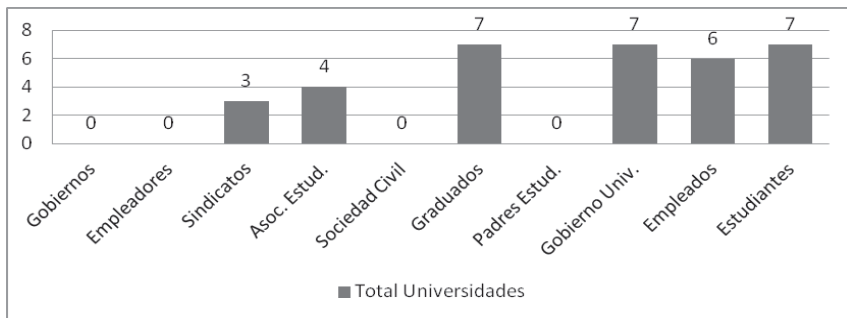

Fuente: Elaboración propia (2010).

Cuadro 3

Partes interesadas universidades de la macro zona norte de Chile

\begin{tabular}{|c|c|c|c|c|c|c|c|c|c|c|}
\hline \multirow[b]{2}{*}{\begin{tabular}{|l} 
Ues Zona \\
Norte de Chile
\end{tabular}} & \multicolumn{7}{|c|}{ Partes interesadas externas } & \multicolumn{3}{|c|}{$\begin{array}{c}\text { Partes interesadas } \\
\text { internas }\end{array}$} \\
\hline & 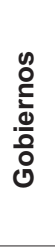 & 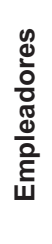 & 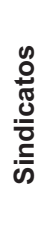 & 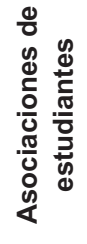 & $\begin{array}{l}\bar{z} \\
\bar{u} \\
\overline{0} \\
\frac{\pi}{0} \\
\frac{\alpha}{U} \\
0 \\
0\end{array}$ & 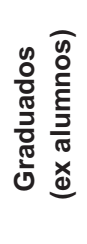 & 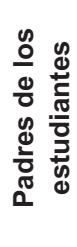 & 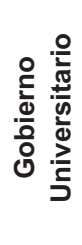 & 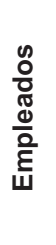 & 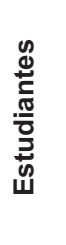 \\
\hline $\begin{array}{l}\text { Universidad } \\
\text { de Tarapacá }\end{array}$ & & & $\mathrm{x}$ & & & $x$ & & $\mathrm{x}$ & $\mathrm{x}$ & $\mathrm{X}$ \\
\hline $\begin{array}{l}\text { Universidad } \\
\text { Arturo Prat }\end{array}$ & & & $X$ & & & $x$ & & $X$ & $X$ & $X$ \\
\hline $\begin{array}{l}\text { Universidad } \\
\text { de Antofagasta }\end{array}$ & $\mathrm{x}$ & & & & & $x$ & & $\mathrm{x}$ & $\mathrm{x}$ & $\mathrm{X}$ \\
\hline $\begin{array}{l}\text { Universidad } \\
\text { Católica del Norte }\end{array}$ & $x$ & & & & & $\mathrm{X}$ & & $\mathrm{x}$ & $x$ & $\mathrm{X}$ \\
\hline $\begin{array}{l}\text { Universidad } \\
\text { de Atacama }\end{array}$ & & & & & & & & $\mathrm{X}$ & $\mathrm{X}$ & $\mathrm{X}$ \\
\hline $\begin{array}{l}\text { Universidad } \\
\text { de La Serena }\end{array}$ & & & & & & $x$ & & $X$ & $x$ & $X$ \\
\hline
\end{tabular}

Fuente: Elaboración propia (2010) con base en la Comisión Europea (2008).

muestra poseen un carácter estatal o público, solamente existiendo el caso de la Universidad Católica del Norte como entidad de carácter privada, la cual además es la más antigua e importante en tamaño dentro de este grupo de universidades.
Como se observa en el Cuadro 3, estas universidades al igual que las de CyL orientan mayoritariamente la estructura de sus sitios de Internet hacia los stakeholders internos, donde el $100 \%$ de las universidades de la MZN posee enlaces 
para dichos grupos, a pesar de que pudiera ser más conveniente la utilización de diseños de intranet para responder a las necesidades de interacción y comunicación con este tipo de partes interesadas, especialmente en lo que se refiere a los empleados y el gobierno universitario.

A su vez, la mayoría de las universidades chilenas de la MZN poseen enlaces para los graduados, o ex alumnos como se denomina en Chile, mientras que del resto de la tipología de la Comisión Europea sólo se registran algunas coincidencias con los sindicatos y el Gobierno, específicamente el Ministerio de Educación (ver Gráfico 2).

Además de los stakeholders de la tipología analizada, existen otras partes interesadas identificadas por las universidades de la MZN de Chile, tales como, futuros alumnos (Tarapacá, Antofagasta), consorcio de universidades del Estado de Chile (Tarapacá, Arturo Prat, Atacama, La Serena), consejo de rectores de las universidades chilenas - $\mathrm{CRUCH}$ - (Arturo Prat, Antofagasta, Atacama, La Serena), proveedores (Ta- rapacá), UNIVERSIA (Tarapacá, Arturo Prat, Antofagasta, Católica del Norte, Atacama, La Serena).

En esta primera comparación entre los sitios web de todas las universidades de la muestra, es posible destacar que existe una mayor convergencia de las universidades de CyL con la tipología analizada, aún cuando la diferencia es leve y se concentra fundamentalmente en los sindicatos y las asociaciones de estudiantes como stakeholders que poseen contenidos en sus sitios web.

Esta diferencia podría ser explicada por el hecho de que en Chile los sindicatos (asociaciones de funcionarios en España) y las asociaciones de estudiantes no cuentan con mayores espacios de participación en el gobierno universitario chileno, por lo que los contenidos y enlaces se dirigen más bien a los estudiantes, docentes y funcionarios de forma individual más que a sus entidades representativas, a diferencia de lo que sucede con las universidades públicas de CyL que si poseen enlaces para dichas instancias.

A su vez, en ambos grupos de universidades se confirma la inexistencia de

\section{Gráfico 2}

\section{Distribución de universidades de la Macro Zona Norte por tipo stakeholder}

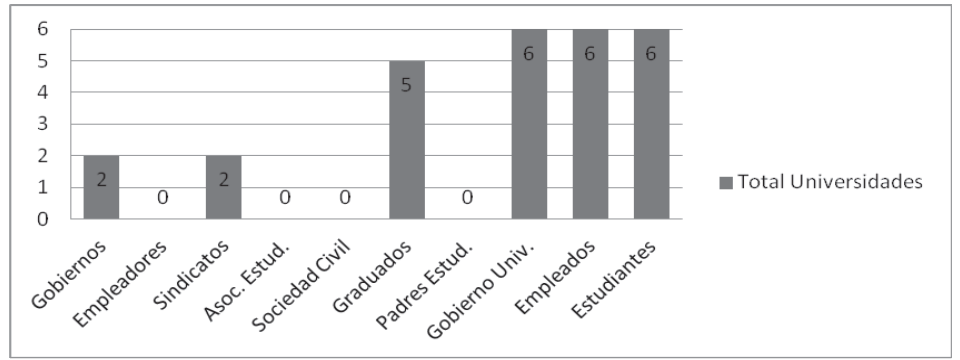

Fuente: Elaboración propia (2010). 
contenidos o enlaces claros y específicos para empleadores, sociedad civil y padres de los estudiantes, lo que conlleva a confirmar que la aplicación a las universidades de tipologías genéricas y restringidas de stakeholders no permite analizar con claridad y precisión la diversidad de partes interesadas con las cuales se relaciona su quehacer universitario, lo que además queda demostrada por la gran cantidad de otros stakeholders que si poseen enlaces o contenidos y que no están presentes en la tipología analizada.

\section{Contenidos de los sitios web de las universidades y relaciones con los stakeholders}

El segundo objetivo de este artículo plantea el análisis de los contenidos utilizados por las universidades en sus sitios web, para relacionarse con las partes interesadas hacia las cuales orientan su quehacer en el espacio virtual.

\section{a) Las Universidades de Castilla} y León (CyL).

Tal y como sucedió con el análisis de los stakeholders, se observan importantes diferencias entre las universidades públicas y las de carácter privado para el caso de CyL, especialmente en los contenidos de procesos estratégicos y comunicación con las partes interesadas. El Cuadro 4 resume los resultados obtenidos del análisis de cada sitio web:

En primera instancia, se observa claramente como las universidades públicas de CyL presentan en sus sitios web un mayor número de los contenidos considerados en este estudio, alcanzando casi las dos terceras partes de los contenidos identificados en sus páginas.
De todas maneras, a pesar de la distribución identificada en el Gráfico 3, resultan especialmente destacables los resultados obtenidos en el caso de la Universidad SEK como institución privada, que incluso se encuentran al mismo nivel de las Universidades de Salamanca y Valladolid, las mejores de la muestra en cuanto a los contenidos puestos a disposición para sus partes interesadas en sus sitios web, de acuerdo al modelo utilizado en este estudio.

El Gráfico 4 presenta la distribución de las universidades antes señalada, destacándose además que las universidades Pontificia de Salamanca y Católica de Ávila ofrecen en sus sitios web poco más de la quinta parte de los contenidos analizados en el presente estudio, 19 en total (Cuadro 4) siendo los más bajos entre todas las instituciones analizadas.

Luego, la revisión de los contenidos de los sitios web de las universidades de CyL muestra un mayor desarrollo en los aspectos relacionados con la información y los procesos electrónicos, siendo menor en los aspectos relacionados con la comunicación con las partes interesadas, que son los contenidos utilizados para mantener un contacto más perma-

\section{Gráfico 3 \\ Contenidos web por tipo de universidad en Castilla y León}

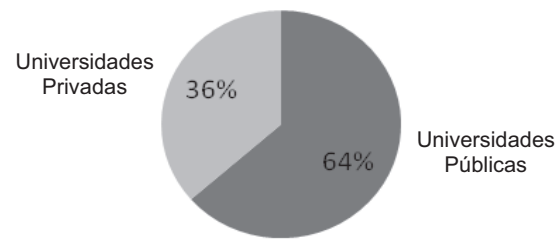

Fuente: Elaboración propia (2010). 


\section{Cuadro 4}

Contenidos de los sitios web en universidades de Castilla y León

\begin{tabular}{|c|c|c|c|c|c|c|c|c|c|c|}
\hline & \multicolumn{4}{|c|}{ Públicas } & \multicolumn{4}{|c|}{ Privadas } & \multirow[b]{2}{*}{ 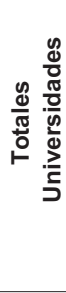 } \\
\hline & & $\begin{array}{l}\text { : } \\
\text { 올 } \\
\overline{0}\end{array}$ & נَّْ & 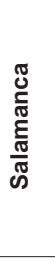 & 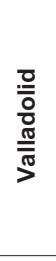 & $\frac{\sqrt[\pi]{2}}{\bar{z}}$ & 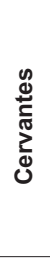 & 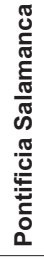 & 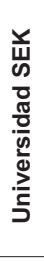 & \\
\hline \multirow{6}{*}{ 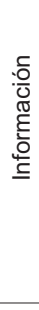 } & $\begin{array}{l}\text { Información financiera (presupuestos, } \\
\text { balances, etc.) }\end{array}$ & & $\mathrm{X}$ & $\mathrm{x}$ & $x$ & & & & & 3 \\
\hline & Certificados de calidad (acreditación) & $\mathrm{x}$ & $x$ & $x$ & $x$ & $\mathrm{x}$ & & & $\mathrm{x}$ & 6 \\
\hline & Becas y ayudas & $x$ & $x$ & $x$ & $x$ & $x$ & $x$ & $x$ & $x$ & 8 \\
\hline & $\begin{array}{l}\text { Mapa o plano localización universidad } \\
\text { (Como llegar) }\end{array}$ & $\mathrm{x}$ & $\mathrm{x}$ & $\mathrm{x}$ & & & $\mathrm{x}$ & $\mathrm{x}$ & $\mathrm{x}$ & 6 \\
\hline & Precios o tasas académicas & $x$ & $x$ & $x$ & $x$ & & & $\mathrm{x}$ & & 5 \\
\hline & Preguntas Frecuentes & $x$ & & $\mathrm{x}$ & $x$ & & $x$ & $x$ & $x$ & 5 \\
\hline \multirow{7}{*}{ 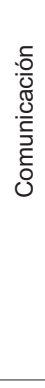 } & Formulario de solicitud de información & & & $x$ & $\mathrm{x}$ & & $x$ & & $\mathrm{x}$ & 4 \\
\hline & $\begin{array}{l}\text { Encuestas de opinión, buzón } \\
\text { de sugerencias o quejas }\end{array}$ & $x$ & $x$ & $\mathrm{x}$ & $\mathrm{x}$ & & & & & 4 \\
\hline & Foros semanales & & & $x$ & & & & & & 1 \\
\hline & $\begin{array}{l}\text { Redes Sociales (propia, Facebook, } \\
\text { Tuenti, Twitter, etc.) }\end{array}$ & & & & $x$ & & & & $x$ & 2 \\
\hline & Blog de la Universidad & & & $x$ & & & & & $x$ & 2 \\
\hline & $\begin{array}{l}\text { Suscripción boletines electrónicos } \\
\text { institucionales }\end{array}$ & $\mathrm{X}$ & & & $x$ & & & & & 2 \\
\hline & Podcast & & & & $x$ & & $x$ & & $\mathrm{x}$ & 3 \\
\hline \multirow{6}{*}{$\begin{array}{l}\text { ") } \\
0 \\
0 \\
0 \\
0 \\
0 \\
0\end{array}$} & Matrículas (online) & $\mathrm{x}$ & & & $x$ & & & & $x$ & 3 \\
\hline & Clases (Campus online) & $x$ & $\mathrm{X}$ & $x$ & $x$ & $\mathrm{X}$ & $x$ & & $x$ & 7 \\
\hline & $\begin{array}{l}\text { Biblioteca virtual (Renovación } \\
\text { préstamos, Revistas Electr.) }\end{array}$ & $x$ & $x$ & $x$ & $\mathrm{x}$ & & $x$ & & $\mathrm{x}$ & 6 \\
\hline & Bolsa de empleos & & & & $x$ & $\mathrm{x}$ & & & $x$ & 3 \\
\hline & Licitaciones & & $x$ & $x$ & $x$ & & & & & 3 \\
\hline & Oposiciones a concursos públicos & & & & & & & & & 0 \\
\hline & Total contenidos & 10 & 9 & 13 & 15 & 4 & 7 & 4 & 12 & \\
\hline
\end{tabular}

Fuente: Elaboración propia (2010).

nente con los stakeholders, mediante foros, chat, blog, redes sociales, entre otros. En cambio, las categorías información y procesos electrónicos responden a necesidades más específicas de la rela- ción de las partes interesadas con las universidades (Gráfico 5).

Como se observa en el Gráfico 5, las universidades Católica de Ávila (comunicación) y Pontificia de Salamanca 


\section{Gráfico 4 Distribución de Contenidos Web por universidad de Castilla y León}

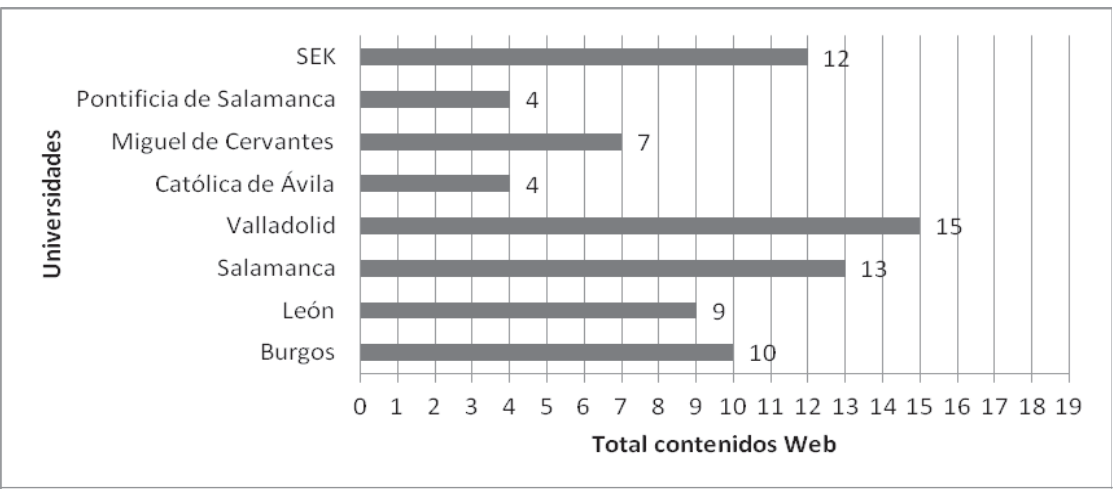

Fuente: Elaboración propia (2010).

\section{Gráfico 5}

\section{Distribución por tipo de contenidos web por universidad de Castilla y León}

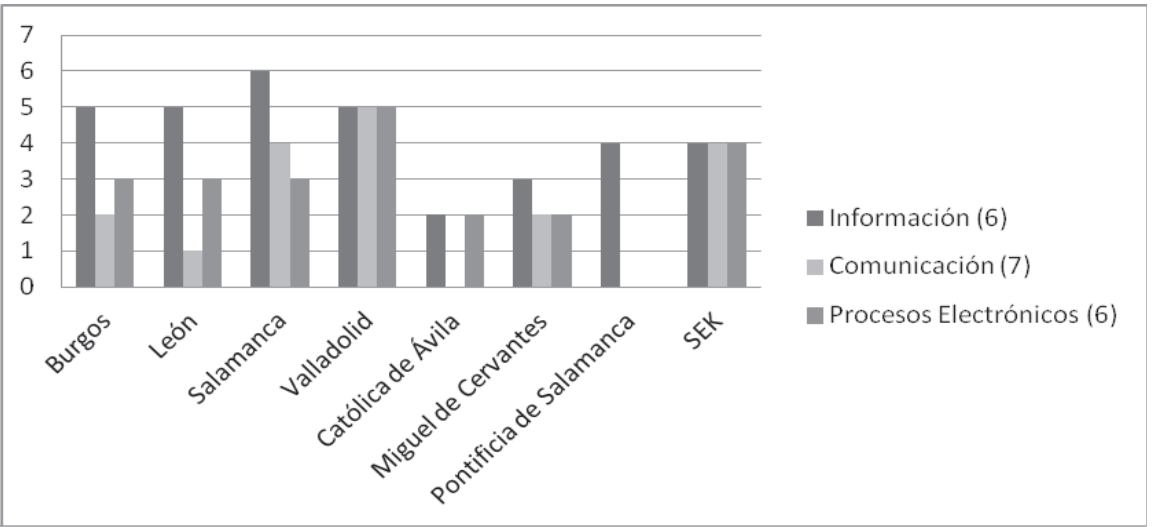

Fuente: Elaboración propia.

(comunicación y procesos electrónicos) son las únicas instituciones de este grupo que no ofrecen contenidos en cada una de las tres categorías analizadas, confirmando con ello la baja capacidad de sus sitios web de transformarse en una plataforma adecuada para el establecimiento de relaciones con sus partes interesadas, cumpliendo un rol mayormente informativo.
En un análisis más detallado, en lo que se refiere a los contenidos sobre información básica, los aspectos referidos a becas y ayudas son los únicos que están presentes en todos los sitios web de las universidades de $\mathrm{CyL}$, mientras que por contrapartida los aspectos relacionados con la información financiera del quehacer institucional sólo está presente en los sitios web de tres universidades de 
Gráfico 6

Distribución de contenido de información por universidades

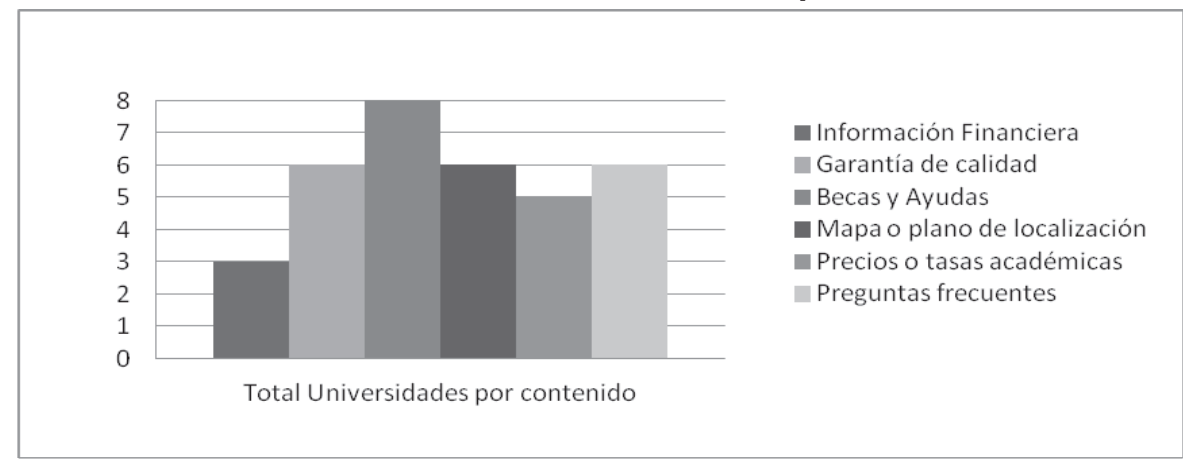

Fuente: Elaboración propia (2010).

Gráfico 7

Total de contenidos web de información por universidades

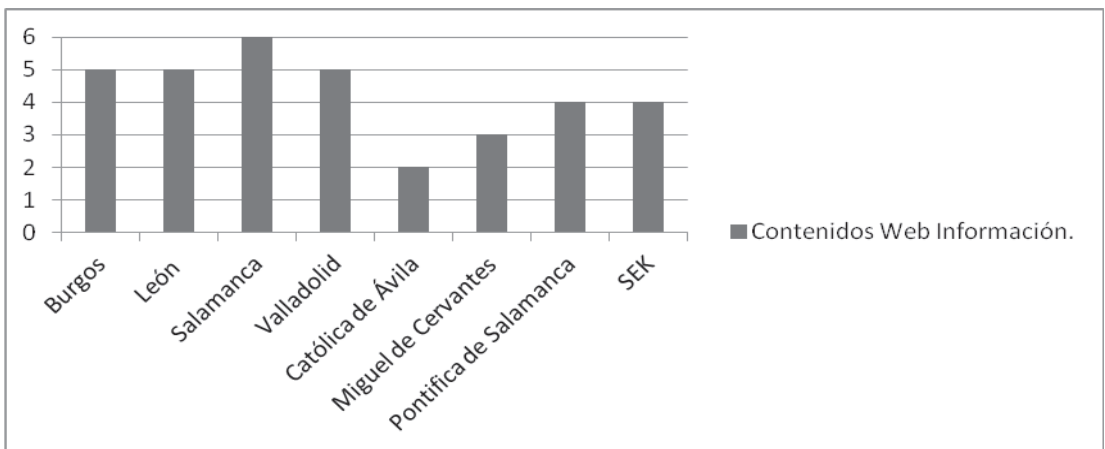

Fuente: Elaboración propia (2010).

la Comunidad (León, Salamanca y Valladolid) (Gráfico 6).

La presencia o ausencia de estos contenidos no sólo debe contemplarse en el plano del acceso a la información básica de cada universidad, sino que también debe analizarse desde la perspectiva del establecimiento de relaciones transparentes, especialmente en el plano contractual o transaccional que es hacia donde mayoritariamente se orientan dichos contenidos, por lo que es importante ob- servar el siguiente gráfico con la distribución de los contenidos por universidad.

El Gráfico 7 nos permite tener presente que es relevante distinguir la importante distorsión que se produce al comparar la información que proveen en Internet las universidades públicas o estatales en comparación con las universidades privadas, dado que si sólo se contrastará los sitios web de las primeras veríamos que todas ellas ofrecen información sobre su acreditación de calidad, becas y ayudas, 
o las tasas académicas, mientras que el $75 \%$ de las universidades públicas de CyL ofrecen enlaces en sus sitios web sobre información financiera (excepto Burgos), plano de localización (excepto Valladolid), preguntas frecuentes (excepto León).

La situación expuesta en el párrafo anterior resulta relevante dado que los contenidos de información y su expedito acceso en Internet se relacionan con el grado de transparencia con la cual las universidades públicas deben establecer su relación con los stakeholders, lo que en muchos casos también se encuentra vinculada a las obligaciones legales que deben cumplir en cuanto al acceso expedito a la información y el escrutinio público.

En segundo término, en cuanto a los aspectos relacionados con los contenidos de comunicación con las partes interesadas de las universidades de CyL, se detectaron aquí los menores desarrollos en los sitios web analizados, observándose los mejores resultados en la Universidad de Valladolid, Salamanca y SEK, tal y como se observa en el Gráfico 8.
A diferencia de la hegemonía de las universidades públicas de CyL en la categoría de información, en esta segunda categoría de análisis la Universidad SEK logra posicionarse en el segundo lugar del grupo, siendo la única universidad de toda la muestra (incluidas las universidades chilenas) que ofrece en su sitio web contenidos y enlaces relativos a redes sociales, blogs y podcast, como expresión del concepto de Web 2.0.

Desde la perspectiva de la distribución por contenidos, los mejores resultados en esta tercera categoría de análisis se obtienen para los formularios de solicitud de información, encuestas, buzón de sugerencias o reclamos, siendo llamativo el bajo desarrollo de los aspectos más activos y permanentes en cuanto a la relación con las partes interesadas, como son las redes sociales, blog y foros de opinión, contenidos habitualmente relacionados con la Web 2.0. Los resultados por contenidos se exponen en el Gráfico 9.

Los enlaces o contenidos de interacción más continua y permanente con los stakeholders, tales como foros temáticos de debate semanal o los blog institu-

\section{Gráfico 8}

\section{Total contenidos web de comunicación por universidades}

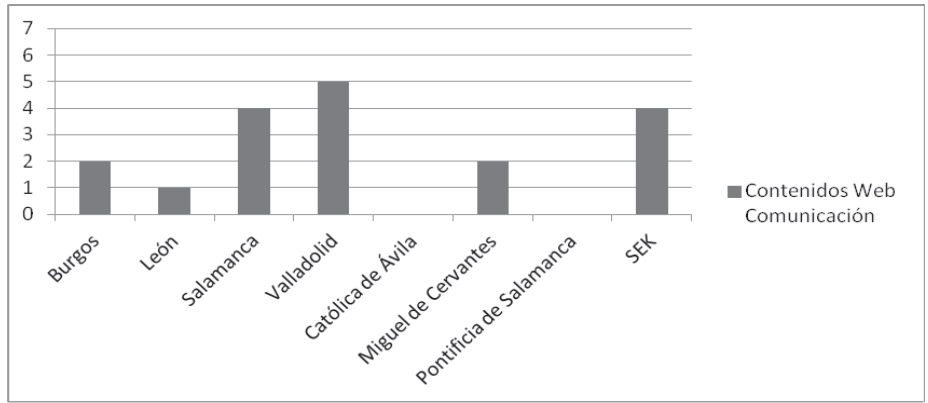

Fuente: Elaboración propia (2010). 


\section{Gráfico 9 \\ Distribución de contenidos web de comunicación por universidades}

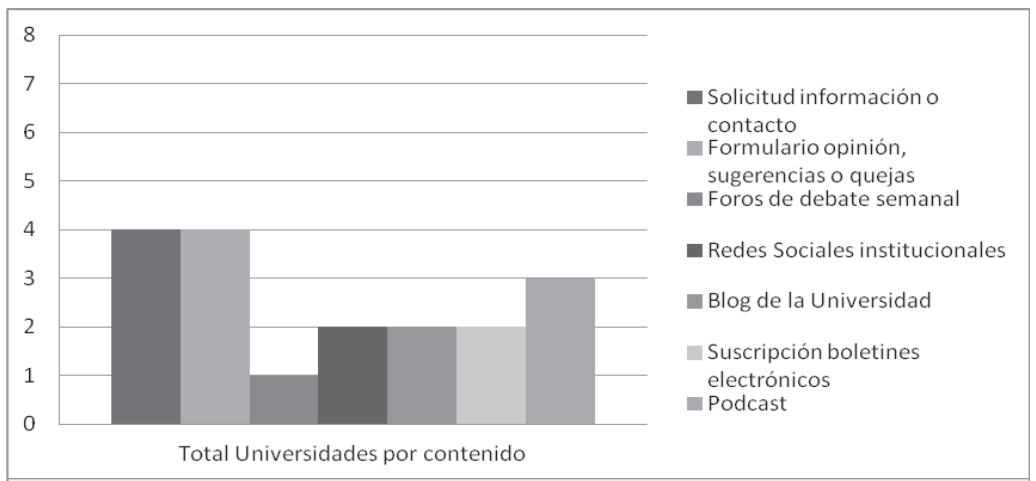

Fuente: Elaboración propia (2010).

cionales registran los resultados más bajos en este segundo grupo de contenidos web, lo que mostraría que la relación de las universidades de CyL con sus partes interesadas se concentran mayormente en el establecimiento de relaciones contractuales o transaccionales, en desmedro del desarrollo de relaciones con los stakeholders con el propósito de afianzar un contacto más permanente y continuo a través del sitio web, posibilitando el establecimiento de redes y comunidades virtuales de aprendizaje e interacción.

Finalmente, en la tercera categoría de análisis utilizada en el presente artículo, los contenidos web relacionados con los procesos estratégicos muestran un desarrollo sólo levemente superior al de los contenidos de comunicación, donde destacan los resultados obtenidos en campus online y biblioteca virtual como los más altos de esta categoría, mientras que no existen contenidos en ninguno de los sitios web de las universidades españolas analizados relativos a los procesos relacionados con las oposiciones (Gráfico 10).
Esta última situación, perfectamente puede ser considerada como un fiel reflejo que denota el excesivo apego a la cultura burocrática con la cual se desarrolla este proceso en la universidad española, que impide virtualizar algunas de las etapas del proceso de oposiciones, especialmente lo referente a las convocatorias y cronogramas. Tampoco deben perderse de vista en este caso, los problemas de endogamia que afectan desde hace un par de décadas a estas universidades (Bermejo, 2009).

En cuanto a la distribución de estos contenidos por universidad, el Gráfico 11 nos permite observar que la Universidad de Valladolid y la Universidad SEK obtienen los mejores resultados, donde esta última institución nuevamente se ubica por sobre el resto de universidades públicas de CyL, mientras que en la otra cara de la moneda la Universidad Pontificia de Salamanca no ofrece en su sitio web ninguno de los contenidos analizados en esta categoría. 


\section{Gráfico 10 \\ Distribución de contenidos web de procesos estratégicos por universidades}

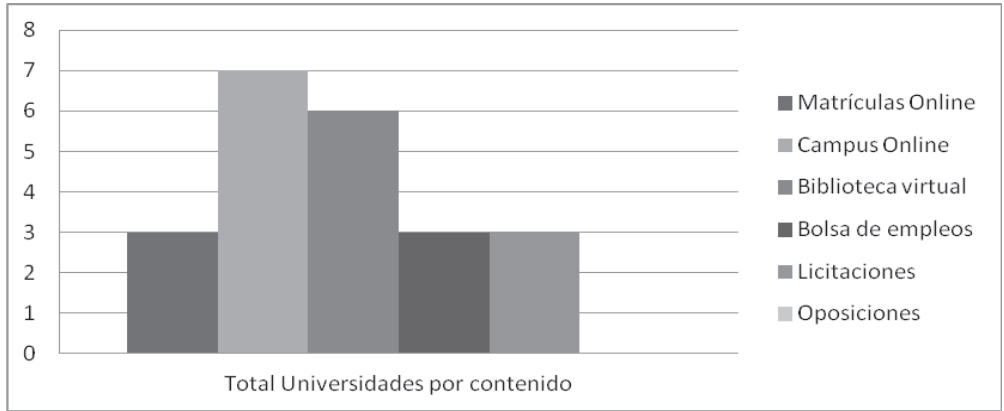

Fuente: Elaboración propia (2010).

\section{Gráfico 11}

\section{Total de contenidos web sobre procesos estratégicos por universidades}

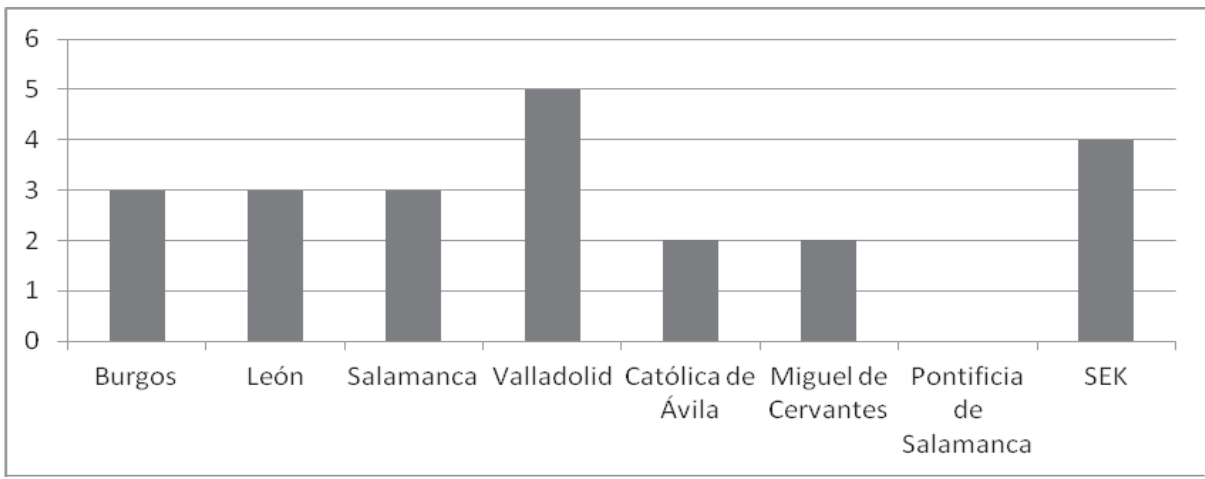

Fuente: Elaboración propia (2010).

De esta manera, los resultados obtenidos para el caso de las universidades de CyL muestran a los contenidos de información y procesos estratégicos como los mejores de esta sub-muestra del estudio, siendo los contenidos de comunicación los que ofrecen los resultados más bajos dentro de este grupo de universidades, especialmente aquellos de carácter más interactivo. b) Universidades macro zona norte de Chile (MZN).

En el caso de estas universidades, a diferencia de las universidades de CyL, sólo la Universidad Católica del Norte es de carácter privada mientras que el resto de instituciones de la MZN de Chile son universidades públicas o estatales, razón por la cual en el análisis de los resultados de este sub-grupo no se hace distinción de este aspecto, como si se realizó en el grupo 
de universidades españolas antes analizado. Los resultados obtenidos para las universidades chilenas se presentan en el Cuadro 5.

Una primera observación que se puede efectuar es que los mejores resultados se obtienen en los contenidos de información, seguidos por los aspectos relacionados con los procesos electrónicos, quedando muy rezagados los contenidos relativos a comunicación, los que poseen muy baja presencia en los sitios web de este grupo de universidades, en algunos casos es totalmente inexistentes, lo que guardando las precisiones de cada caso permite observar una similitud entre ambos grupos de universidades analizadas.

Como se observa en el Gráfico 12, las universidades de Antofagasta y de Atacama se transforman en las únicas universidades de la sub-muestra que no poseen contenidos en sus sitios Web para la categoría de comunicación, lo que sería indicativo de sitios web de carácter informativo y estáticos que sólo permiten establecer relaciones uni-direccionales.

De manera más detallada, en el caso de los contenidos de información destaca el hecho de que todas las universidades de este grupo informen sobre su situación financiera, acreditación de calidad y respecto de becas y ayudas, quedando sólo un escalón por debajo lo relacionado con información sobre precios o aranceles. Con un desarrollo más bajo, aparecen los aspectos más específicos como el plano de ubicación de la universidad o las preguntas frecuentes. Los resultados de esta categoría se presentan en el Gráfico 13.
Respecto a lo anterior, es importante mencionar que por efectos de la Ley de Transparencia y Acceso a la Información $N^{0} 20.285$, que afecta a las universidades estatales en su condición de servicios públicos, les obliga a publicar en sus sitios web diversas informaciones relacionadas con la dotación de personal o los recursos financieros y presupuestarios asignados.

También, respecto a la acreditación de calidad por imperativo de Ley 20.129 que establece el Sistema Nacional de Aseguramiento de la Calidad de la Educación Superior, exige que todas las universidades estatales deben acreditarse e informar de acuerdo a los años y áreas que mantienen acreditadas, razón por la cual los sitios web se transforman en una plataforma de información ideal para dichos propósitos de transparencia de la información.

Un comentario adicional que merece el Gráfico 13, se refiere a los resultados obtenidos para el mapa o plano de localización y las preguntas frecuentes, las que aún cuando parecieran secundarias, para el caso de estas universidades de carácter regional y de un tamaño menor dentro del concierto chileno, se transforman en elementos que pueden ayudar a las nuevas partes interesadas para poder contactarlas, por ejemplo los nuevos estudiantes.

En cuanto a la distribución de los contenidos de la categoría información por universidad de la MZN de Chile, como se observa en el Gráfico 14 las universidades Católica del Norte y de La Serena poseen en sus sitios web todos los elementos analizados (6), no obstante todas las universidades de este grupo poseen más del $50 \%$ de los contenidos de esta categoría. 


\section{Cuadro 5 \\ Contenidos de los sitios web en universidades de la Macro Zona Norte de Chile}

\begin{tabular}{|c|c|c|c|c|c|c|c|c|}
\hline & & 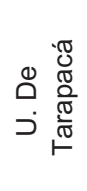 & 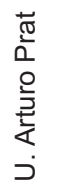 & 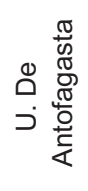 & 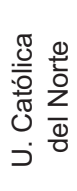 & 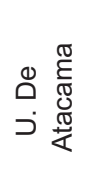 & 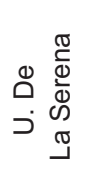 & 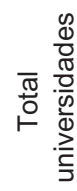 \\
\hline \multirow{6}{*}{ 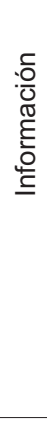 } & $\begin{array}{l}\text { Información financiera } \\
\text { (presupuestos, balances, etc.) }\end{array}$ & $x$ & $x$ & $x$ & $x$ & $x$ & $x$ & 6 \\
\hline & $\begin{array}{l}\text { Certificados de calidad } \\
\text { (acreditación) }\end{array}$ & $x$ & $x$ & $x$ & $x$ & $x$ & $x$ & 6 \\
\hline & Becas y ayudas & $\mathrm{x}$ & $\mathrm{x}$ & $x$ & $\mathrm{x}$ & $\mathrm{x}$ & $x$ & 6 \\
\hline & $\begin{array}{l}\text { Mapa o plano localización } \\
\text { universidad (Como llegar) }\end{array}$ & & $x$ & & $x$ & & $x$ & 3 \\
\hline & $\begin{array}{l}\text { Precios o tasas académicas } \\
\text { (aranceles) }\end{array}$ & $x$ & & $x$ & $x$ & $x$ & $x$ & 5 \\
\hline & Preguntas Frecuentes & & $x$ & & $x$ & & $x$ & 3 \\
\hline \multirow{7}{*}{ 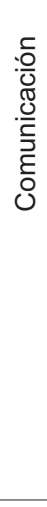 } & $\begin{array}{l}\text { Formulario de solicitud de } \\
\text { información o contacto }\end{array}$ & & $x$ & & $x$ & & $x$ & 3 \\
\hline & $\begin{array}{l}\text { Encuestas de opinión, buzón de } \\
\text { sugerencias o quejas }\end{array}$ & & $x$ & & $x$ & & & 2 \\
\hline & $\begin{array}{l}\text { Foros semanales abiertos a la } \\
\text { comunidad }\end{array}$ & & & & & & & 0 \\
\hline & $\begin{array}{l}\text { Redes Sociales (propia, Facebook, } \\
\text { Tuenti, Twitter, etc.) }\end{array}$ & $x$ & & & & & & 1 \\
\hline & Blog de la Universidad & & & & & & & 0 \\
\hline & $\begin{array}{l}\text { Suscripción boletines electrónicos } \\
\text { institucionales }\end{array}$ & & & & & & $x$ & 1 \\
\hline & Podcast & $x$ & & & & & & 1 \\
\hline \multirow{6}{*}{ 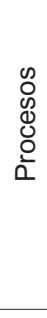 } & Matrículas (online) & & $x$ & $x$ & $x$ & $x$ & $x$ & 5 \\
\hline & Clases (Campus online) & & $x$ & & & & & 1 \\
\hline & $\begin{array}{l}\text { Biblioteca virtual (Renovación } \\
\text { préstamos, Revistas Electrónicas) }\end{array}$ & $x$ & $x$ & $x$ & $x$ & $x$ & $x$ & 6 \\
\hline & Bolsa de empleos & $\mathrm{x}$ & $\mathrm{x}$ & $x$ & & & $x$ & 4 \\
\hline & Licitaciones & & & & & & & 0 \\
\hline & Oposiciones a concursos públicos & & $x$ & $x$ & $\mathrm{x}$ & & & 3 \\
\hline & Total contenidos & 8 & 12 & 8 & 11 & 6 & 11 & \\
\hline
\end{tabular}

Fuente: Elaboración propia (2010). 


\section{Gráfico 12}

Distribución por tipo de contenidos Web por universidades

\section{de la Macro Zona Norte de Chile}

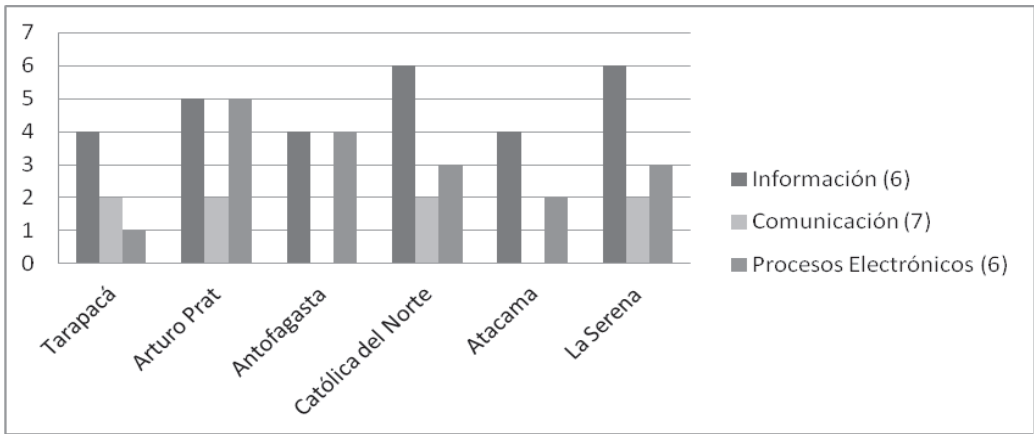

Fuente: Elaboración propia (2010).

\section{Gráfico 13}

\section{Distribución de contenido de información por universidades}

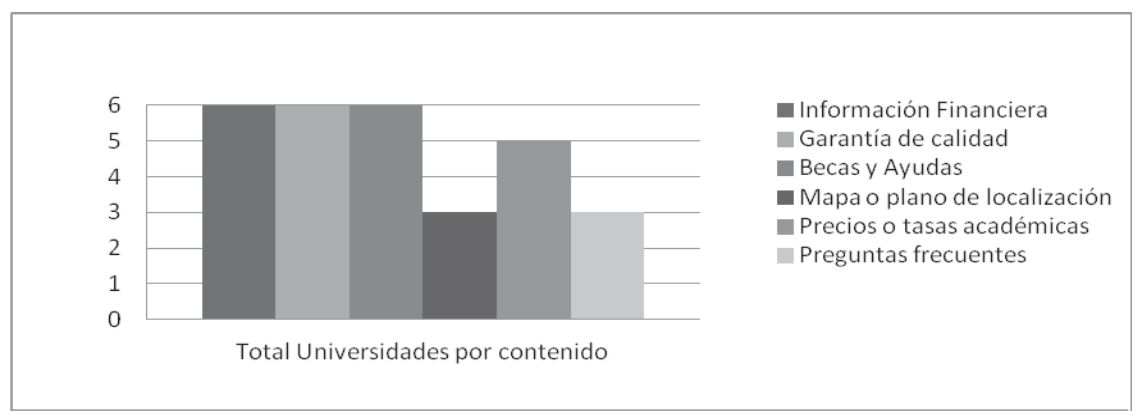

Fuente: Elaboración propia (2010).

\section{Gráfico 14}

Total de contenidos web de información por universidades

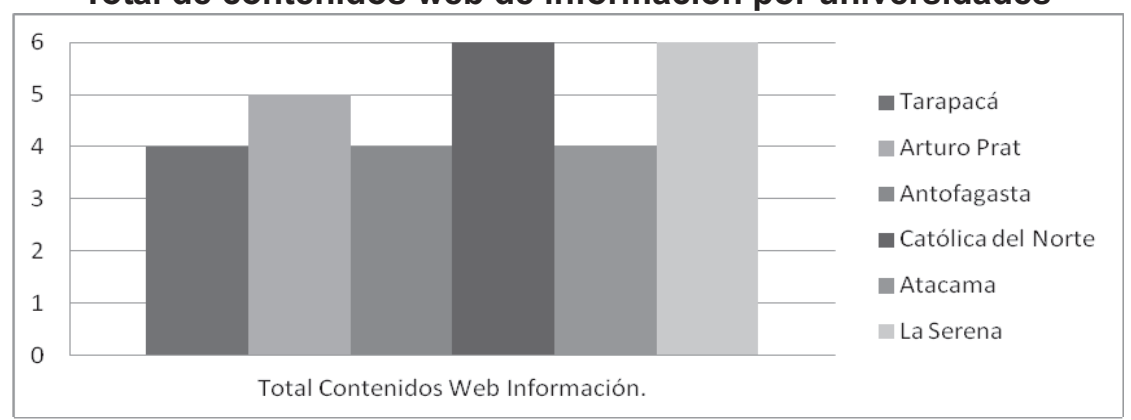

Fuente: Elaboración propia (2010). 


\section{Gráfico 15 \\ Distribución de contenidos web de procesos estratégicos por universidades}

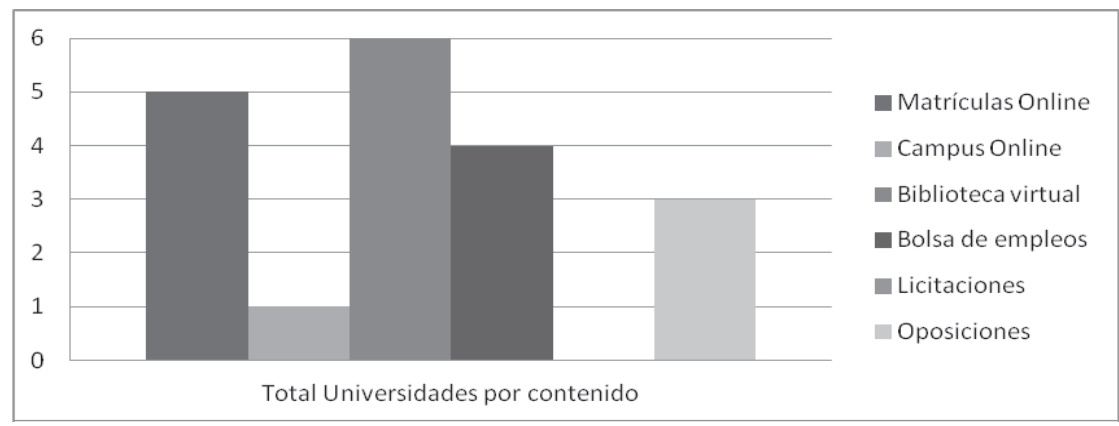

Fuente: Elaboración propia (2010).

Sin embargo, es importante destacar el caso de la Universidad Católica del Norte, única universidad privada de este grupo como se señaló anteriormente, y que posee todos los contenidos de esta categoría, a pesar de no tener las obligaciones legales de transparencia y acceso a la información que si afectan a las universidades estatales chilenas.

En segundo lugar, en lo que se refiere a los contenidos de procesos estratégicos, se observa un desarrollo menor y dispar entre las universidades de la MZN de Chile, exceptuando lo relacionado con los servicios online de la biblioteca donde todas las universidades de este grupo ofrecen en sus sitios web enlaces y contenidos sobre este ámbito.

El otro contenido que presenta un importante desarrollo en los sitios web de estas universidades es la Matrícula online, donde sólo la Universidad de Tarapacá no ofrece enlaces claros relacionados con este proceso, lo cual no quiere decir que no lo desarrolle a través de Internet, y que probablemente debido al carácter es- tacional de este procesos no se encontraban activos en el sitio web en el período de realización de este estudio.

Respecto del Gráfico 15, también es importante observar el bajo desarrollo de lo que se refiere al campus online, donde sólo la Universidad Arturo Prat ofrece contenidos claros y específicos sobre este aspecto, lo que sin lugar a dudas no sólo resta posibilidades de establecer mejores relaciones con los stakeholders, sino que además resta oportunidades a las universidades de la macro zona norte para ofrecer más servicios académicos, así como respecto de sus posibilidades de internacionalización e interacción con partes interesadas más allá de sus fronteras geográficas.

A lo anterior, se suma la inexistencia de contenidos relacionados con las licitaciones públicas que puedan demandar estas instituciones en su relación con los proveedores, a pesar de que algunas de ellas mantienen en sus sitios web enlaces con el sitio Chile Compra que centraliza las compras públicas de los servi- 
cios públicos en Chile, lo que podría considerarse como sustitutivo de enlaces relativos a las licitaciones públicas.

En cuanto a la distribución de los contenidos de procesos estratégicos por universidades, como se observa en el Gráfico 16 ninguna de las universidades de la MZN posee todos los elementos de esta categoría, siendo la Universidad Arturo Prat y la Universidad de Antofagasta las que ofrecen los mejores resultados, siendo las únicas que además superan el $50 \%$ de los contenidos de esta categoría, mientras que en el otro extremo la Universidad de Tarapacá presenta el menor desarrollo en esta categoría, ofreciendo enlaces y contenidos solamente para la biblioteca virtual y la bolsa de trabajos.

Finalmente, los contenidos relacionados con la comunicación con las partes interesadas ofrecen el desarrollo más bajo dentro de este grupo de universidades, donde el contenido con mayor presencia es el enlace para completar la solicitud de información o contacto, seguido por los enlaces para el formulario de opinión, sugerencias o quejas.
Además, dos de las universidades de la MZN (Antofagasta y Atacama) no presenta enlaces para ninguno de los contenidos de comunicación, tal como se mostrara anteriormente en el Gráfico 12. Los resultados por contenido de comunicación se presentan en el Gráfico 17.

Los resultados obtenidos en este último grupo de contenidos, permiten inferir la existencia de una muy baja interacción virtual de las universidades de la macro zona norte con sus stakeholders, y un claro desaprovechamiento de las ventajas y oportunidades que ofrecen las nuevas tecnologías de información y comunicación para establecer relaciones más fluidas y permanentes con estos grupos, profundizando el tipo de relación de carácter contractual o transaccional que las organizaciones establecen con ellos.

De esta manera, considerando los resultados de ambos grupos de universidades (españolas y chilenas) es posible confirmar la mayor presencia de los contenidos web relacionados con los aspectos de Información, y en menor medida respecto de los contenidos asociados

\section{Gráfico 16}

\section{Total contenidos web sobre procesos estratégicos por universidades}

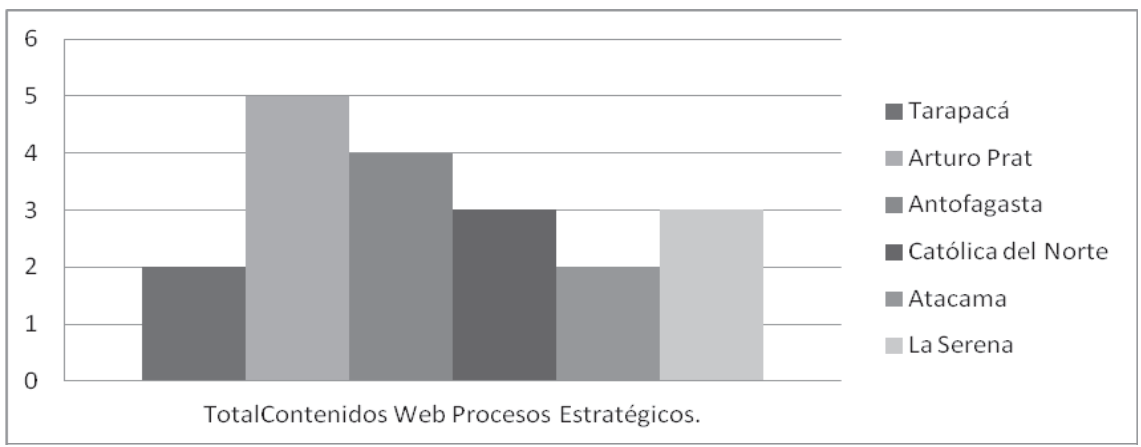

Fuente: Elaboración propia (2010). 
Gráfico 17

Total de contenidos web de comunicación por universidades

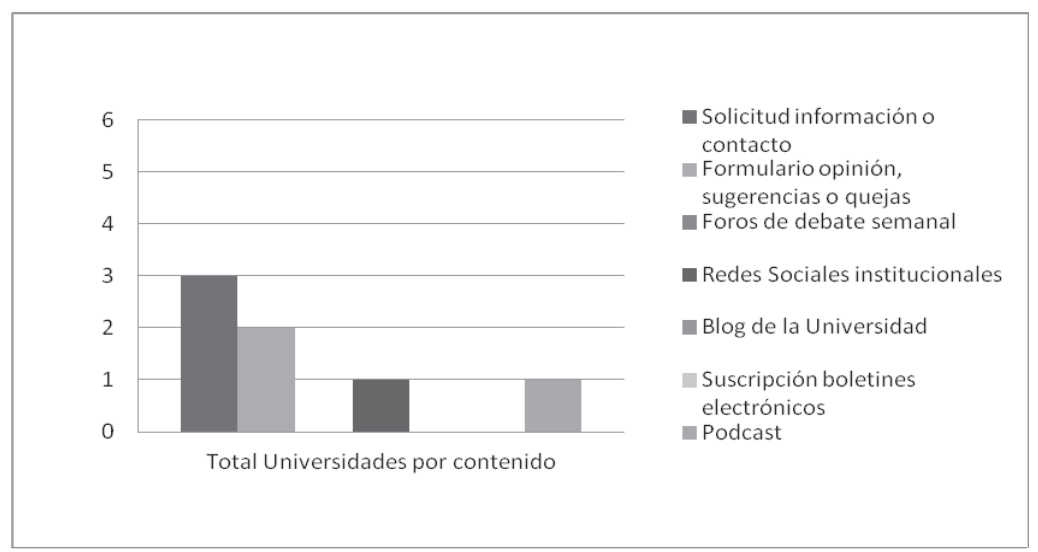

Fuente: Elaboración propia.

con la comunicación y los procesos estratégicos.

c) Similitudes y diferencias entre los sitios web de las universidades españolas y chilenas de la muestra.

En primer lugar, como fue señalado anteriormente, tanto las universidades españolas como chilenas presentan sus mejores resultados en los contenidos web de información y procesos estratégicos, siendo los aspectos relacionados con la comunicación con las partes interesadas el ámbito menos desarrollado en sus sitios web, especialmente en lo que se refiere a foros de debate semanal abiertos a la comunidad y blog institucionales, situación que es especialmente débil en el caso de las universidades chilenas de la muestra. EI Gráfico 18 permite observar esta situación.

A través de este gráfico se constata como las universidades españolas de VaIladolid y SEK son, con diferente frecuencia, las instituciones que poseen un desa- rrollo y presencia más equilibrada de las categorías analizadas en sus sitios web. La categoría información, es la única que ofrece universidades con todos los contenidos analizados en la misma (6), en este caso las universidades Católica del Norte y de La Serena en Chile y la Universidad de Salamanca en España.

Sin embargo, a nivel más detallado el único contenido que está presente en todos los sitios web consultados es la información sobre becas y ayudas, el que sin dudas desde una perspectiva del marketing se transforma en un elemento de importante "atracción" para el reclutamiento de los nuevos estudiantes que se matriculan en cada universidad, por sobre los contenidos relativos a la certificación de calidad o la información de precios o tasas académicas.

En contraposición, el contenido "foros semanales" es el que está menos presente entre todas las universidades analizadas (sólo Salamanca), lo que se trans- 


\section{Gráfico 18}

\section{Distribución de contenidos web por categoría y universidades}

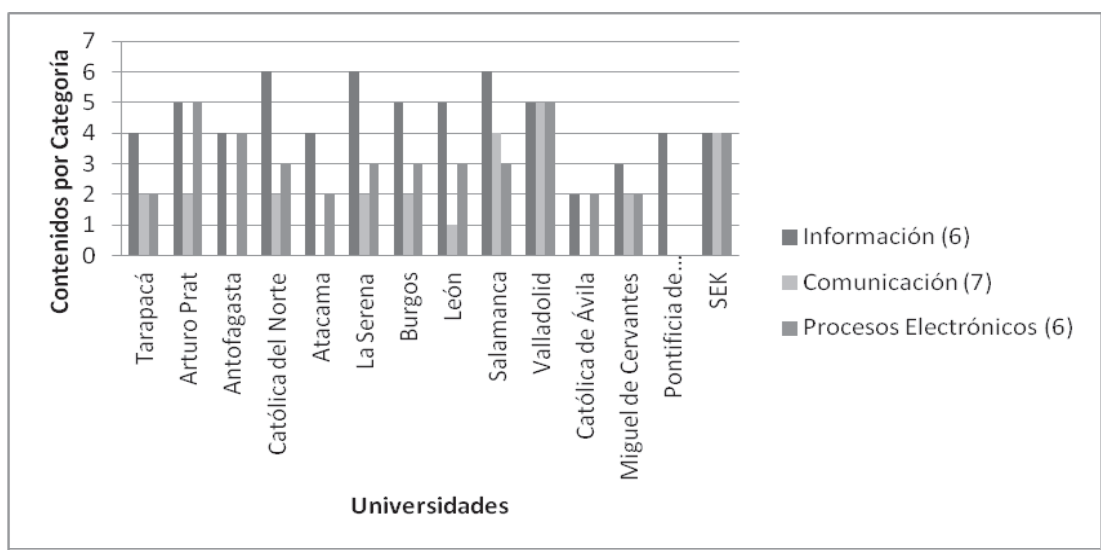

Fuente: Elaboración propia (2010).

forma en la "punta del iceberg" del bajo desarrollo de los contenidos de comunicación, que como se ha señalado anteriormente son indicativos de relaciones más constantes, interactivas y reticulares con los stakeholders.

Otro ámbito en donde se observan importantes similitudes es en la baja presencia o utilización de elementos de la Web 2.0 en los sitios de Internet analizados, que para el caso de las universidades de la macro zona norte de Chile es especialmente débil, sólo existiendo el caso de la Universidad de Tarapacá que posee enlaces hacia una red social así como el uso de podcast, no existiendo ningún ejemplo para el caso de los blogs institucionales.

La situación anterior permite reforzar la percepción respecto del bajo desarrollo de relaciones interactivas y de carácter reticular con las partes interesadas, siendo dominante en ambos grupos de universidades las relaciones de carácter contractual o transaccional.
Una diferencia importante se produce en relación con el contenido "oposiciones", único que no está presente en ninguna de las universidades españolas analizadas en la muestra, y que en el caso de las universidades chilenas está presente en un $50 \%$. Como se señaló anteriormente, una posible explicación de esta situación es que estos procesos en España se encuentran en manos de los Departamentos en cada Facultad, existiendo importantes acusaciones de endogamia entorno a estos procesos, lo que podría explicar la nula presencia de información o enlaces en los sitios web de cada universidad.

\section{Conclusiones}

Una primera conclusión de este artículo se relaciona con la identificación de los stakeholders de una organización, y los criterios para realizar dicho proceso: (1) dichos actores responden a sectores de actividad y misiones institucionales 
específicas, más allá de las clasificaciones genéricas disponibles en la literatura; (2) se encuentran tanto al interior como al exterior de la organización; (3) cada stakeholder tiene un interés o se ve afectado de manera diferente, no siempre coincidente con los demás grupos de interés; (4) variarán en sus niveles de interés por participar en el proceso de toma de decisiones; y (5) la diversidad de partes interesadas permiten desarrollar redes sociales para aumentar la creación de riqueza de sus miembros.

En términos generales, al analizar las tipologías genéricas y extendidas obtenidas desde la literatura, se observa la existencia de un núcleo convergente de partes interesadas que se identifican en las mismas: empleados, clientes, accionistas, gobiernos, proveedores y comunidad local. Con excepción de esta última, los otros cinco stakeholders se muestran más cercanos a un carácter contractual de su relación con una organización.

Otra conclusión se relaciona con la identificación de las partes interesadas a quienes las universidades de CyL dirigen los contenidos de sus sitios web, donde además de las partes interesadas en la tipología de la Comisión Europea (sindicatos, titulados, asociaciones de estudiantes, gobierno universitario, empleados y estudiantes), destacan los preuniversitarios, las fundaciones universitarias y algunas empresas con las cuales se mantiene una relación específica, como las principales partes interesadas de esas universidades.

En el caso de las universidades chilenas de la MZN, existe la misma tendencia hacia los stakeholders internos y los ex alumnos, al igual como en el caso de las universidades españolas de la mues- tra. No obstante, en el caso de las universidades chilenas no existen enlaces o contenidos para las organizaciones de representación de empleados y estudiantes, debido a que éstos no pertenecen al gobierno universitario.

En cuanto a los contenidos de los sitios web, tanto las universidades de CyL como de las universidades de la MZN de Chile estructuran las relaciones virtuales con sus partes interesadas en base a los aspectos relacionados con la información y los procesos electrónicos, lo que puede interpretarse como una mayor orientación hacia las relaciones de carácter contractual y uni-direccional con sus stakeholders, en lugar de aquellas dirigidas hacia el establecimiento de relaciones más complejas de carácter reticular, que permiten una mayor generación de riqueza para la comunidad de partes interesadas donde la organización asume un rol de liderazgo nodal.

De esta manera, la importancia e impacto que puede tener el uso de elementos de la Web 2.0 en el fortalecimiento de las relaciones con sus partes interesadas puede ser determinante para el desarrollo futuro de estas universidades, permitiéndoles incluso establecer un radio de acción más amplio para el establecimiento de relaciones con otras partes interesadas que se transformen en socios estratégicos en dicho crecimiento.

Para finalizar, este tipo de estudios permiten a las universidades reconocer buenas prácticas que les ayuden a fortalecer las relaciones con sus stakeholders, dimensionando de mejor manera el potencial que su sitio web puede ofrecer, no sólo con un carácter informativo sino que además en cuanto 
al establecimiento de una plataforma para desarrollar su quehacer en el siglo XXI, caracterizado por el uso intensivo de las nuevas tecnologías de la información y la comunicación, ámbito en el cual ya incursionan hace varios años las grandes empresas y las universidades norteamericanas.

\section{Referencias bibliográficas}

Agle, Bradley; Ronald Mitchell \& Jeffrey Sonnenfled (1999). Who matters to CEOs? An investigation of stakeholder attributes and salience, corporate performance, and CEO values, Academy of Management Journal, 42(5): 507-525.

Antonacopoulou, Elena \& Jérôme Meric (2005). From power to knowledge relationships: Stakeholder interactions as learning partnerships, pp.125-147, en Stakeholder Theory. A European perspective, eds. Bonnafous-Boucher, M. \& Pesqueux, Y, PalgraveMacmillan: New York.

Bermejo, José Carlos (2009). La fábrica de la ignorancia. La universidad del como si, Ediciones Akal, Madrid España.

Burrows, Joanne (1999). Going beyond labels: a framework for a profiling institutional stakeholder, en Contemporary Education, 70(4):5-10.

Caballero, G., García, J.M., \& Quintás, M.A. (2007). La importancia de los stakeholders de la organización: un análisis empírico aplicado a la empleabilidad del alumnado de la universidad española, en Revista Investigaciones Europeas de Dirección y Economía de la empresa, 13(2):13-32.

Caballero, G., Vázquez, X.H. \& Quintás, M.A. (2009). ¿Qué influencia tienen los stakeholders de la universidad española en la empleabilidad de sus alumnos?
Propuestas de reforma, en Cuadernos de economía y dirección de empresa, №38, marzo, pp.37-64. $<$ http://www.acede.org/fotos/pdf/art 248_38_02.pdf> [31 de enero de 2010]

Carroll, Archie (1991). The pyramid of corporate social responsibility: toward the moral management of organizational stakeholders, Business Horizons, 34(4):30-48.

Clarkson, Max (1995). A stakeholder framework for analyzing and evaluating corporate social performance, Academy of Management Review, 20(1): 92-117.

Comisión Europea (2008). Higher Education Governance in Europe. Policies, structures, funding and academic staff, Eurydice: Brussels.

Comisión Europea (2001), El libro Verde: Fomentar un marco europeo para la responsabilidad social de las empresas, Bruselas Bélgica.

Donaldson, Thomas \& Lee Preston (1995). The stakeholder theory of the corporation: Concepts, evidence and implications, Academy of Management Review, 20(1): 65-91.

Durán, Juan José (2006). Economic and social responsibility of the multinational enterprise, pp. 379-392, en Economy, entrepreneurship, science and society in the XXI century, eds. Del Val, M., Sánchez, Y. \& García, C., Universidad de Alcalá: Alcalá de Henares.

Ferrary, Michel (2005). A stakeholder perspective of human resource management, pp. 104-124, en Stakeholder Theory. A European perspective, eds. Bonnafous-Boucher, M. \& Pesqueux, Y. Palgrave-Macmillan: New York.

Freeman, R. Edward (1984). Strategic Management: A stakeholder approach, Pitman: Massachusetts. 
Gaete, Ricardo (2010). Discursos de responsabilidad social universitaria: el caso de las universidades de la macro zona norte de Chile pertenecientes al Consejo de Rectores, Perfiles Educativos, 32(128):27-54. [Acceso: 19 de mayo de 2010] http://www.scielo.org.mx/pdf/peredu/v32n128/v32n 128a3.pdf

Hax, Herbert (2006). The role of entrepreneurial ethics in a market economy, pp. 393-404, en Economy, entrepreneurship, science and society in the XXI century, eds. Del Val, M., Sánchez, Y. \& García, C., Universidad de Alcalá: Alcalá de Henares España.

Lozano, Josep (1999). Ética y empresa, Trotta: Madrid.

Lozano, Josep (2009). La empresa ciudadana como empresa responsable y sostenible, Trotta: Madrid.

Marín, Francisco (2008). Responsabilidad social corporativa y comunicación, Fragua: Madrid.

Matten, Dirk, Andrew Crane \& Wendy Chapple (2003). Behind the mask: revealing the true face of corporate citizenship, Journal of Business Ethics, 45(1-2): 109-120.

Moneva, José Mariano (2005). Información sobre responsabilidad social corporativa: Situación y tendencias, Revista Asturiana de Economía RAE, 34: 43-67.
Post, James; Lee Preston \& Sybille Sachs (2002a). Managing the extended enterprise: the new stakeholder view, California Management Review, 45(1): 6-28.

Post, James; Lee Preston \& Sybille Sachs (2002b). Redefining the corporation. Stakeholder management and organizational wealth, California: Stanford University Press.

Prandi, María (2007). La gestión de los derechos humanos en la empresa, Revista de Estudios Sociales y de Sociología aplicada, 146: 111-127.

Setó, Dolores (2007). Una nueva actitud de la empresa hacia su entorno: La responsabilidad social, Revista Alta Dirección, Año XLII, 251/252: 49-57.

Sison, Alejo José (2008). Corporate Governance and Ethics. An Aristotelian perspective, Cheltenham: Edward Elgar Publishing.

Torres, Enrique (2006). The corporate responsibility, pp. 467-475, en Economy, entrepreneurship, science and society in the XXI century, eds. Del Val, M., Sánchez, Y. \& García, C., Universidad de Alcalá: Alcalá de Henares.

Werther, William \& Chandler, David (2006). Strategic corporate social responsibility. Stakeholders in a global environment, SAGE publications, California EE.UU. 